\title{
Produção de fatores de virulência in vitro por espécies patogênicas do gênero Candida
}

\author{
Production of virulence factors in vitro by pathogenic species \\ of the genus Candida
}

Kelly Cristina Ortolan Rörig ${ }^{1}$, Jean Colacite ${ }^{1}$ e Maxwel Adriano Abegg ${ }^{1}$

\begin{abstract}
RESUMO
Avaliou-se, in vitro, a capacidade de crescimento em $39^{\circ} \mathrm{C}$ e $42^{\circ} \mathrm{C}$, a produção de enzimas hidrolíticas e a atividade hemolítica de 21 cepas clínicas e de referência de sete espécies de Candida spp, Candida dubliniensis e Candida krusei demonstraram menor potencial de virulência e Candida albicans maior.
\end{abstract}

Palavras-chaves: Candida spp. Fatores de virulência. Enzimas hidrolíticas. Fungos patogênicos.

\section{ABSTRACT}

The growth capacity at $39^{\circ} \mathrm{C}$ and $42^{\circ} \mathrm{C}$, production of hydrolytic enzymes and hemolytic activity of 21 clinical and reference strains of seven species of Candida spp were evaluated in vitro. Candida dubliniensis and Candida krusei demonstrated lower virulence potential and Candida albicans higher potential.

Key-words: Candida spp. Virulence factors. Hydrolytic enzymes. Pathogenic fungi.

Leveduras do gênero Candida são frequentemente comensais humanos, mas podem, em situações que normalmente envolvem imunodebilidade, causar infecção conhecida como candidíase ou candidose, em diversos sítios anatômicos ${ }^{2}$.

Estas micoses podem ser causadas por diferentes espécies. Candida albicans continua sendo a espécie mais prevalente, mas espécies de Candida não-albicans, particularmente Candida tropicalis, Candida glabrata, Candida krusei e Candida parapsilosis, tem adquirido importância crescente ${ }^{16}$.

Por consequiência, há interesse nos fatores de virulência destas leveduras, para estabelecer estratégias de controle com antifúngicos e prevençã $0^{411}$.

A habilidade de produzir enzimas hidrolíticas é considerada um importante fator de virulência ${ }^{5}$. As principais enzimas produzidas por leveduras do gênero Candida são as proteinases e as fosfolipases ${ }^{13}$.

Diferentes espécies de Candida demonstram atividade hemolítica quando crescidas em ágar-sangue enriquecido ${ }^{78}$.

Outra característica associada com a patogenicidade em humanos é a propriedade de multiplicação a altas temperaturas, como $39^{\circ} \mathrm{C}$ e $42^{\circ} \mathrm{C}^{6}$.

1. Curso de Farmácia, Universidade Paranaense, Toledo, PR.

Endereço para correspondência: Prof. Maxwel Adriano Abegg. Rua Jurandir DalPrá,

548 apto 12, Vila Becker, 85902-530 Toledo, PR

Tel: 5545 3054-1813

e-mail: maxwel@unipar.br

Recebido para publicação em 17/03/2008

Aceito em 05/03/2009
O objetivo deste trabalho foi avaliar o potencial de virulência in vitro de 21 cepas clínicas e de referência de sete espécies de Candida.

Organismos testados. Foi investigado um isolado de referência e dois isolados clínicos de sete espécies (Candida albicans, Candida tropicalis, Candida parapsilosis, Candida glabrata, Candida krusei, Candida guilliermondii e Candida dubliniensis) de Candida. Os isolados foram identificados por avaliação morfológica (micromorfologia em ágar fubá-tween 80) e por testes bioquímicos através do Kit Candifast (International Microbio) e API 20 C (bioMérieux).

Pesquisa de enzimas hidrolíticas. A produção de fosfolipase foi verificada utilizando-se o método do ágar gema de ovo em placa, de acordo com Kantarcioglu e Yucel ${ }^{4}$. As placas de Petri foram incubadas a $37^{\circ} \mathrm{C}$ e os diâmetros das colônias e das colônias mais zonas de precipitação foram mensuradas após 7 dias de incubação. A zona de atividade de fosfolipase $(\mathrm{Pz})$ foi calculada de acordo com Price cols ${ }^{9}$, em termos do índice do diâmetro da colônia dividido pelo diâmetro da colônia mais a zona de precipitação. A produção de proteinase foi evidenciada através de halos claros em torno das colônias, em placas de ágar contendo albumina de soro bovina (BSA), conforme Kantarcioglu e Yucel ${ }^{4}$. $O$ índice Pz foi determinado da mesma forma que para a fosfolipase.

A atividade de amilase foi testada com meio contendo 3\% de amido de batata solúvel, com pH ajustado a 5, de acordo com Tsuyoshi cols ${ }^{15}$. Os halos de atividade foram revelados como zonas claras em torno das colônias pela adição de lugol. 
A produção de gelatinase foi verificada em placa contendo $0,8 \%$ de gelatina e $5 \%$ de ágar. Uma alçada de cada isolado foi inoculada na superfície do ágar e este foi incubado a $30^{\circ} \mathrm{C}$ por 7 dias. A produção de gelatinase fica evidente pela formação de halos claros em torno das colônias, utilizando ácido tricloroacético como revelador, conforme Kanemitsu cols ${ }^{3}$.

Crescimento a $39^{\circ} \mathrm{C}$ e $42^{\circ} \mathrm{C}$. Para isto, $10 \mu \mathrm{L}$ de caldo Sabouraud com turvação ajustada ao tubo 1 da escala de Mac Farland, obtido de células novas dos isolados, foi inoculado em ágar Sabouraud-dextrose e incubado a $39^{\circ} \mathrm{C} \mathrm{e} 42^{\circ} \mathrm{C}$ por $72 \mathrm{~h}$, de acordo com Llanos cols ${ }^{6}$.

Atividade hemolítica. Foram utilizadas placas de Ágar Sabouraud dextrose contendo $3 \mathrm{~mL}$ de hemácias de sangue humano lavadas 3 vezes com tampão salina-fosfato (PBS) ${ }^{8}$. Discos de papel filtro com $6 \mathrm{~mm}$ de diâmetro foram posicionados em pontos equiidistantes na placa e inoculados com $10 \mu \mathrm{L}$ de cultivo das células em caldo Sabouraud com turvação ajustada ao tubo 1 da escala de Mac Farland. Todos os testes foram realizados em triplicata para cada isolado.

A Tabela 1 mostra que os isolados de Candida albicans produziram mais proteinase e fosfolipase que as demais espécies. Esta observação concorda com os resultados de Silva cols ${ }^{13}$, que, comparando a produção enzimática de Candida albicans $\left(\mathrm{n}^{0}=31\right)$ e Candida parapsilosis $\left(\mathrm{n}^{\mathrm{0}}=6\right)$, observaram que $100 \%$ dos isolados de Candida albicans foram produtores de proteinase e 83,8\% de fosfolipase. Kantarcioglu e Yucel $^{4}$, estudando a produção de fosfolipase e proteinase em 95 isolados clínicos de várias espécies de Candida (Candida albicans, Candida glabrata, Candida guilliermondii, Candida kefyr, Candida krusei, Candida lipolytica, Candida lusitaniae, Candida parapsilosis, Candida rugosa e Candida tropicalis), igualmente observaram que Candida albicans apresentou um percentual significativamente maior de isolados produtores de proteinase e fosfolipase.

Linares cols ${ }^{5}$ estudaram a atividade enzimática e capacidade hemolítica de Candida dubliniensis $\left(\mathrm{n}^{\mathrm{0}}=18\right)$ frente à Candida albicans $\left(\mathrm{n}^{\mathrm{0}}=30\right)$. Semelhante ao observado em nosso estudo, claramente Candida albicans é maior produtora de fosfolipase e proteinase e também apresenta maior capacidade hemolítica que esta outra espécie.

Tamura cols ${ }^{14}$ avaliaram a produção de fatores de virulência (hidrofobicidade da superfície celular, aderência, atividades de proteinase e de fosfolipase) em 23 amostras de: Candida albicans $\left(\mathrm{n}^{\mathrm{0}}=7\right)$, Candida tropicalis e Candida parapsilosis $\left(\mathrm{n}^{\mathrm{0}}=6\right)$ cada, e Candida lusitaniae e Candida glabrata $\left(\mathrm{n}^{0}=2\right)$. A maior atividade de fosfolipase e de proteinase (Pz menor) foi de Candida parapsilosis isolada de cateter venoso central. A espécie Candida albicans apresentou atividade levemente inferior (Pz maior) à de

\section{TABELA 1}

Resultados dos testes de atividade de fosfolipase, proteinase, amilase, crescimento em $42^{\circ} \mathrm{C}$ e $39^{\circ} \mathrm{C}$ e atividade hemolítica em 21 isolados de Candida spp.

\begin{tabular}{|c|c|c|c|c|c|}
\hline \multirow[b]{2}{*}{ Isolados } & \multirow[b]{2}{*}{ Proteinase } & \multirow[b]{2}{*}{ Fosfolipase $(\mathrm{Pz})$} & \multicolumn{3}{|c|}{ Crescimento } \\
\hline & & & Amilase & $42^{\circ} \mathrm{C} / 39^{\circ} \mathrm{C}$ & Hemólise \\
\hline Candida albicans ATCC 18.804 & 0,36 & 0,34 & + & $+/+$ & ++++ \\
\hline Candida albicans UEM 1 & 0,42 & 0,38 & + & $+/+$ & +++ \\
\hline Candida albicans UFRGS 51 & 1,0 & 0,37 & + & $+/+$ & ++ \\
\hline Candida tropicalis ATCC 13.803 & 1,0 & 0,46 & + & $+/+$ & - \\
\hline Candida tropicalis UFRGS 55 & 0,43 & 1,0 & + & $+/+$ & - \\
\hline Candida tropicalis UFRGS 56 & 1,0 & 0,48 & + & $+/+$ & - \\
\hline Candida glabrata ATCC 2.001 & 1,0 & 1,0 & + & $+/+$ & - \\
\hline Candida glabrata UEM 24 & 1,0 & 1,0 & + & $+/+$ & - \\
\hline Candida glabrata UEM 118 & 1,0 & 1,0 & + & $-/+$ & - \\
\hline Candida guilliermondii ATCC 46.036 & 1,0 & 1,0 & + & $+/+$ & - \\
\hline Candida guilliermondii UEM 28 & 1,0 & 1,0 & + & $+/+$ & - \\
\hline Candida guilliermondii UEM 73 & 1,0 & 1,0 & + & $+/+$ & - \\
\hline Candida krusei ATCC 6258 & 1,0 & 1,0 & + & $-/+$ & - \\
\hline Candida krusei SM 1 & 1,0 & 1,0 & - & $+/+$ & - \\
\hline Candida krusei SM 2 & 1,0 & 1,0 & + & $-/+$ & - \\
\hline Candida dubliniensis CBS 7987 & 0,44 & 1,0 & + & $-1+$ & - \\
\hline Candida dubliniensis SM 23 & 0,43 & 1,0 & - & $-/-$ & - \\
\hline Candida dubliniensis SM 25 & 0,44 & 1,0 & - & $-/$ & - \\
\hline Candida parapsilosis ATCC 22.019 & 0,42 & 1,0 & + & $+/+$ & ++ \\
\hline Candida parapsilosis UEM 115 & 1,0 & 1,0 & + & $+/+$ & + \\
\hline Candida parapsilosis UEM 81 & 1,0 & 1,0 & - & $+/+$ & + \\
\hline
\end{tabular}

+ : positivo, - : negativo, ++++ : muito fortemente positivo, +++ : fortemente positivo, ++ : positivo, + : fracamente positivo, Pz: índice de atividade de fosfolipase. 1,0: isolado inativo. ATCC: American Type Culture Collection, EUA, UEM: Universidade Estadual de Maringá, PR, UFRGS: Universidade Federal do Rio Grande do Sul, RS, SM: Universidade Federal de Santa Maria, RS, CBS: Centraalbureau voor Schimmelcultures, Holanda. 
Candida parapsilosis. De fato, neste estudo Candida albicans não se destacou como a maior produtora destas enzimas, sendo sua produção até levemente inferior à de Candida tropicalis e Candida glabrata.

Estudando espécies de Candida quanto à atividade amilásica, Tsuyoshi cols ${ }^{15}$ não observaram atividade amilásica em Candida glabrata. Rosa cols ${ }^{10}$ não observaram atividade de amilase em cinco espécies de Candida avaliadas (Candida albicans, Candida tropicalis, Candida krusei, Candida parapsilosis, Candida guilliermondii).

Nos testes feitos a $42^{\circ} \mathrm{C}$, observou-se que somente os isolados de Candida dubliniensis e dois isolados de Candida krusei não obtiveram resultados positivos. Já em $39^{\circ} \mathrm{C}$, que é uma temperatura de pacientes febris, observou-se que todos os isolados de Candida dubliniensis não apresentaram crescimento, isto significa que pacientes imunodebilitados, com temperatura a $39^{\circ} \mathrm{C}$ podem desenvolver candidíase por certas espécies do gênero. Esta sensibilidade de Candida dubliniensis a temperaturas elevadas parece responder em parte pela sua reduzida capacidade invasiva, mesmo em pacientes severamente imunodebilitados.

Somente os isolados de Candida parapsilosis e Candida albicans apresentaram atividade hemolítica neste estudo.

Diferentemente, Luo cols ${ }^{7}$ não observaram capacidade hemolítica em cinco isolados de Candida parapsilosis avaliados, enquanto reportaram atividade hemolítica em outras espécies. Igualmente neste estudo Candida albicans demonstrou ter maior capacidade hemolítica que as demais espécies. Os autores usaram sangue de carneiro, enquanto que neste estudo foi utilizado sangue humano na preparação do meio de cultura. O sangue humano ou o processamento dos cultivos de Candida podem ser fatores que dificultam a manifestação da capacidade hemolítica por espécies que não Candida albicans.

Esta atividade de enzimas hidrolíticas elevada de Candida albicans está de acordo com sua maior virulência em infecções sistêmicas $i$ in vivo ${ }^{10}$. No entanto, Samaranayake cols ${ }^{11}$, avaliando a expressão de fosfolipase B em 30 isolados de Candida albicans, usando placas e teste em caldo, de pacientes infectados pelo vírus da imunodeficiência humana (HIV), não observaram associação significativa da produção desta enzima com outros quatro fatores de virulência avaliados.

\section{REFERÊNCIAS}

1. Candido CR, Azevedo PVR, Komesu CM. Enzimotipagem de espécies do gênero Candida isolados da cavidade bucal. Revista da Sociedade Brasileira de Medicina Tropical 33: 437-442, 2000.

2. Fonzi A, Calderone RA. Virulence factors of Candida albicans. Trends in Microbiology 9: 327-335, 2001.

3. Kanemitsu K, Nishino T, Kunishima H. Quantitative determination of gelatinase activity among enterococci. Journal of Microbiological Methods 47: 11-16, 2001

4. Kantarcioglu AS, Yucel A. Phospholipase and protease activity in clinical Candida isolates with reference to the sources of strains. Mycoses 5: 160-165, 2002.

5. Linares CEB, Loreto ES, Silveira CP, Pozzatti P, Scheid LA, Santurio JM, Alves SH. Enzymatic and hemolytic activities of Candida dubliniensis strains. Revista do Instituto de Medicina Tropical de São Paulo 49: 203-206, 2007.

6. Llanos R, Fernandez-Espinar MT, Querol A. A comparison of clinical and food Saccharomyces cerevisiae isolates on the basis of potential virulence factors. Antoine Van Leuwenhoek 90: 221-231, 2006.

7. Luo G, Samaranayake LP, Yau JYY. Candida species exhibit differential in vitro hemolytic activities. Journal of Clinical Microbiology 39: 2971-2974, 2001.

8. Manns JM, Mosser DM, Buckley HR. Production of a hemolytic factor by Candida albicans. Infection and Immunity 62: 5154-5156, 1994.

9. Price MF, Wilkinson ID, Gentry LO. Plate methods for detection of phospholipase activity in Candida albicans. Sabouraudia 20: 7-14, 1982.

10. Rosa EAR, Pereira CV, Rosa RT, Hofling JF. Grouping oral Candida species by multilocus enzyme electrophoresis. International Journal of Systematic and Evolutionary Microbiology 50: 1343-1349, 2000.

11. Samaranayake YH, Dassanayake RS, Jayatilake JAMS, Cheung BPK, Yau JYY, Yeung KWS, Samaranayake LP. Phospholipase B enzyme expression is not associated with other virulence attributes in Candida albicans isolates from patients with human immunodeficiency virus infection. Journal of Medical Microbiology 54: 583-593, 2005.

12. Schaller M, Borelli C, Korting, HC, Hube B. Hydrolytic enzymes as virulence factors of Candida albicans. Mycoses 48: 365-377, 2005.

13. Silva JO, Ferreira JC, Candido R.C. Atividade enzimática, produção de slime e sensibilidade a antifúngicos de Candida sp. Revista da Sociedade Brasileira de Medicina Tropical 40: 354-355, 2007.

14. Tamura NK, Negri MFN, Bonassoli LA, Svidzinski TIE. Fatores de virulência de Candida spp. isoladas de cateteres venosos e mãos de servidores hospitalares. Revista da Sociedade Brasileira de Medicina Tropical 40: 91-93, 2007.

15. Tsuyoshi N, Fudol R, Yamanaka S. Identification of yeast strains isolated from marcha in sikkin, a microbial started for amylolytic fermentation. International Journal of Food Microbiology 99: 135-146, 2005.

16. Yang YL. Virulence factors of Candida species. Journal of Microbiology Immunology and Infection 36: 223-228, 2003. 